\title{
CONVERGENCE, CLOSED PROJECTIONS AND COMPACTNESS
}

\author{
J. E. VAUGHAN ${ }^{1}$
}

ABSTRACT. In this paper several results of N. Noble concerning closed projections are extended using generalizations of first countable, Fréchet, and sequential spaces. We also consider compactness conditions defined by requiring that certain nets have cluster points.

1. Introduction. The three concepts mentioned in the title are related in some interesting ways. As is well known, a space $X$ is compact if and only if for every space $Y$, the projection $\pi_{Y}: X \times Y \rightarrow Y$ is a closed map. In [3] it was proved that a space $X$ is countably compact if and only if for every sequential space $Y$ the projection $\pi_{Y}: X \times Y \rightarrow Y$ is closed. In [13], N. Noble was interested in generalizing this and related results by use of $[a, b]$-compactness and $b-a$-sequential spaces, and he gave some ingenious techniques for studying the relations among these concepts. Several theorems in Noble's paper [13] are stated in terms of [a, b]-compactness, but the property "every $b$-a-sequence has a cluster point" is used in the proofs instead of $[a, b]$-compactness. We noted in [17] that this property (which we called property $N[a, b]$ ) is not equivalent to $[a, b]$-compactness, and so several results in [13] need to be reconsidered. Theorem 1.4 of [13] should be restated in terms of $N[a, b]$ rather than $[a, b]$-compactness. When this is done, Theorem 1.4 of [13] says that every $b$-a-sequence in $X$ has a cluster point if and only if for every $\mathfrak{b}$-a-sequential space $Y$, the projection $\pi_{Y}$ : $X \times Y \rightarrow Y$ is closed. This and similar results suggest that there is a connection between (a) certain nets in $X$ have cluster points, (b) the topology of $Y$ is "determined" by the same kind of nets, and (c) the projection $\pi_{Y}: X \times Y \rightarrow Y$ is closed. This fact is the content of Theorem 1 in this paper.

Our main result (Theorem 2 ) deals with infinite product spaces, and its

Received by the editors May 20, 1974.

AMS (MOS) subject classifications (1970). Primary 54A20, 54B10; Secondary 54D20, 54D55.

Key words and phrases. Sequential, Fréchet, and first countable spaces, Q-net, §-F réchet, and $\Omega$-neighborhood spaces, closed projections on finite and infinite product spaces, compact, countably compact and initially $m$-compact spaces, $\mathbf{Q}$ compact spaces.

1 This research was partially supported by a grant from the University of North Carolina Research Council, Greensboro, North Carolina. 
proof is based on the techniques developed by Noble to prove Theorem 4.1 of [13]. We pointed out in [16] that Theorem 4.1 of [13] is not correctly stated, and we gave a version of it there. In this paper we get another version of it as a corollary to Theorem 2 .

2. Convergence and closed projections. We will use the standard terminology concerning convergence of nets (Moore-Smith sequences) as found in [11]. Let $\Omega$ be a class of directed sets. A net $f$ is called an $\Omega$-net if its domain is in $\Omega$. We define cardinal numbers to be initial ordinal numbers, and denote them by $\omega_{\mu}$. In particular $\omega=\omega_{0}$ and $\omega_{1}$ are respectively the first infinite and the first uncountable ordinals.

Definition 1. A collection of subsets $\mathcal{F}$ of a set $X$ is said to be an $\Omega$ filter base ( $\Omega$ is.a class of directed sets) if there exists $D \in \Omega$ and a map $f$ of $D$ onto $\mathcal{F}$ such that $d \leq d^{\prime}$ implies $f(d) \supseteq f\left(d^{\prime}\right)$.

Definition 2. A topological space $X$ is called an $\Omega$-neighborhood space provided each point $x \in X$ has a local base which is an $\Omega$-filter base.

Definition 3. A space $X$ is called an $\Omega$-Fréchet space provided that for every $H \subset X$, and every $x \in \bar{H}$, there exists an $\Omega$-net with values in $H$ which converges to $x$.

Definition 4. A space is called an $\Omega$-net space provided a set $H \subseteq X$ is closed if and only if $f$ is an $\Omega$-net with values in $H$ which converges to $x$ implies $x$ is in $H$.

What we have called an $\Omega$-Fréchet space was called a space of "type $\subseteq \Omega$ " by G. Birkhoff [2] and a $ß$-space by K. Wichterle in closure spaces [18]. When $\Omega=\{\omega\}$, these definitions reduce to their prototypes: first countable, Fréchet, and sequential spaces (see S. P. Franklin [4]). Situations similar to the one above have been considered by several authors. P. R. Meyer [14] has considered the case where $\Omega$ is the class of all directed sets having cardinality less than or equal to m. H. Herrlich [7], N. Howes [9], and D. C. Kent [12] have considered the class of all well-ordered sets, and D. Harris [6] has considered the case where $\Omega=\left\{\omega_{\mu}\right\}$ and $\omega_{\mu}$ is regular.

Definition 5. For a space $X$ and an $\Omega$-net $f: D \rightarrow X$ which converges to $x \in X$, let $W(f, D, x)=f(D) \cup\{x\}$. A space $X$ is said to have the $\Omega$-weak topology provided a subset $H \subset X$ is closed if and only if $H \cap W(f, D, x)$ is closed in each $W(f, D, x)$ with respect to the subspace topology on $W(f, D, x) \subset X$.

It is well known that for the case $\Omega=\{\omega\}$, Definitions 4 and 5 are equivalent. We give an example at the end of this section to show that they are not the same for every $\Omega$. For a space $X$, these $W(f, D, x)$ form a natural cover in the sense of Franklin [5]. 
Definition 6. A space $X$ is said to be $\Omega$-compact if every $\Omega$-net in $X$ has a cluster point.

It is easy to check that a space is $\Omega$-compact if and only if every $\Omega$ filter base $\mathcal{F}$ on $X$ has an adherent point (i.e., $\bigcap\{\bar{F} \mid F \in \mathcal{F}\} \neq \varnothing$ ), and that many kinds of compactness are special cases of $\Omega$-compactness for a suitable choice of the class $\Omega$.

If $\Omega$ is the class of all directed sets, then $\Omega$-compactness is just compactness. If $\Omega=\{\omega\}$, then a space is $\Omega$-compact if and only if it is countably compact. A space $X$ is called initially $m$-compact if every filter base $\mathcal{F}$ on $X$ with $|\mathcal{F}| \leq \mathfrak{m}$ has an adherent point. If $\Omega$ is the class of all directed sets having cardinality $\leq \mathrm{m}$, then $\Omega$-compactness is equivalent to initial m-compactness. If $\Omega$ is the class of all directed sets $D$ such that every countable subset of $D$ has an upper bound in $D$, then $\Omega$-compactness is equivalent to the Lindelöf property. If $\Omega$ is the class of all $<a$-directed sets $D$ (i.e., every subset of $D$ of cardinality strictly less than $a$ has an upper bound) with $|D| \leq \mathfrak{b}$, then $\Omega$-compactness is by definition the property $G[a, b]$ discussed in [18]. If $\Omega$ is the set of all $S_{a}(m)=\{H \mid H \subset m$ and $|H|<a\}$ where $a \leq \mathfrak{m} \leq \mathfrak{b}$, then $\Omega$-compactness is, by definition, the property $N[a, b]$ mentioned in $\bar{\S}_{1}$. If $\Omega=\left\{\omega_{\mu}\right\}$ where $\omega_{\mu}$ is regular, then $\Omega$-compactness was called $\omega_{\mu}$-compactness in [6].

Theorem 1. Let $X$ be a topological space and $\Omega$ a class of directed sets.

A. The following five conditions are equivalent.

1. For every $\Omega$-net space $Y, \pi_{Y}: X \times Y \rightarrow Y$ is a closed map.

2. For every subspace $Y$ of an $\Omega$-net space, $\pi_{Y}: X \times Y \rightarrow Y$ is a closed map.

3. For every $\Omega$-Fréchet space $Y, \pi_{Y}: X \times Y \rightarrow Y$ is a closed map.

4. For every $\Omega$-neighborhood space $Y, \pi_{Y}: X \times Y \rightarrow Y$ is a closed map.

5. $X$ is $\Omega$-compact.

B. The next condition implies each of the above five.

6. For every space $Y$ with the $\Omega$-weak topology, $\pi_{Y}: X \times Y \rightarrow Y$ is a closed map.

Proof. The only implications which require much effort are $4 \rightarrow 5$ and $5 \rightarrow 1$, and these are simple extensions of Mrówka's proof of the analogous situation for sequences [15, p. 4]. More general results of this nature are known [4]. The definition of $\Omega$-net space was chosen to make the proof of 
the implication $5 \rightarrow 1$ as easy as possible. Finally, $6 \rightarrow 1$ because every $\Omega$-net space has the $\Omega$-weak topology (see Proposition 1 ).

We now consider some other ways that one might have used to define the concept of $\Omega$-net spaces.

Definition 7. Let $a$ be an ordinal number and $\Omega$ a class of directed sets: We now define the ath $\Omega$-net closure operator for a space $X$. It will be denoted by $S_{\alpha}^{\Omega}$ or by $S_{a}$ if the class $\Omega$ is understood. For each $H \subset X$ define $S_{0}(H)=H$ and $S_{\alpha}(H)=\bigcup\left\{S_{\beta}(H) \mid \beta<\alpha\right\}$ if $\alpha$ is a limit ordinal and $S_{\alpha}(H)=$ $S_{\tau}(H) \cup\left\{x \in X \mid x\right.$ is the convergent limit of an $\Omega$-net in $\left.S_{\tau}(H)\right\}$ where $\tau$ is the immediate predecessor of $a$.

This concept was defined for the sequential case by A. Arhangel'skii and S. Franklin [1], by P. Meyer for the $m$-sequential case [14], and Wichterle [18] for closure spaces. Any one of the following seven conditions, which are equivalent for the sequential case, $\Omega=\{\omega\}$, would be a candidate for the definition of $\Omega$-net space. We chose the first one as the definition because it was convenient in Theorem 1. It is interesting that six of these conditions are equivalent for any class $\Omega$ of directed sets. The proof of this fact is essentially the same as the proofs of various results in the theory of sequential spaces and closure spaces. We sketch a proof for convenience of the reader.

Proposition 1. For a space $X$ with topology $\mathcal{T}$, conditions (i)-(vi) below are equivalent and imply condition (vii). Let $H \subset X$.

(i) $H$ is closed iff $f$ is an $\Omega$-net with values in $H$ and $f$ converges to $x$ implies $x$ is in $H$.

(ii) For every $H \subset X, \bar{H}=S_{a}(H)$ for some ordinal a (note that the $\Omega$ Fréchet spaces are those spaces such that $\left.\bar{H}=S_{1}(H)\right)$.

(iii) $H$ is closed iff $f$ is an $\Omega$-net which converges to $x$ and $f$ is frequently in $H$ implies $x$ is in $H$.

(iv) $H$ is closed iff $f$ is an $\Omega \cdot n e t$ which converges to $x$ and $x \notin H$ implies $f$ is eventually in $X \backslash H$.

(v) $U$ is open iff $f$ is an $\Omega$-net which converges to $x \in U$ implies $f$ is eventually in $U$.

(vi) The topology $\mathcal{T}$ for $X$ is the largest for which each $\mathcal{T}$-convergent $\Omega$-net converges. By this we mean the following: If $\mathcal{J}^{\prime}$ is a topology for $X$ which satisfies the condition:

(*) If $f$ is an $\Omega$-net and $\mathcal{T}$-converges to $x$, then $f$ also $\mathcal{J}^{\prime}$-converges to $x$, then $\mathcal{T}^{\prime} \subseteq \mathcal{T}$.

(vii) $X$ has the weak topology with respect to convergent $\Omega \cdot n e t s$. 
Proof. (i) $\rightarrow$ (ii). Set $r=|X|^{+}$, i.e., $\tau$ is the first initial ordinal larger than the cardinal number of $X$. Since $H \subset S_{\tau}(H) \subset \bar{H}$, one can easily prove the desired result by showing that $S_{\tau}(H)$ is a closed set.

(ii) $\rightarrow$ (iii). One can easily prove by transfinite induction that $S_{\alpha}(H)=H$ for all $\alpha$. The result follows from this. (iii) $\rightarrow$ (iv) and (iv) $\rightarrow$ (v) are trivial, and (v) $\rightarrow$ (vi) is straightforward.

(vi) $\rightarrow$ (i). This result follows easily from the following observation:

Lemma. If $f: D \rightarrow X$ is a net which converges to $x$ and is frequently in $H \subset X$, then there exists a net $g: D \rightarrow H$ such that $g$ converges to $x$.

We have shown that (i) to (vi) are equivalent. It is easy to prove that (i) $\rightarrow$ (vii) and this completes the proof.

We now show that (vii) is not equivalent to the other six properties in Proposition 1 .

Lemma. Let $\Omega$ be a class of directed sets with the property that for each $D \in \Omega$, every subset of $D$ with cardinality less than $m$ has an upper bound in $D$ (i.e., $D$ is <m-directed). If $X$ is a $T_{1}, \Omega$-net space, then either $X$ is a discrete space or the character of $X$ is greater than or equal to $\mathrm{m}$.

Proof. We assume that $X$ is not discrete and show that $X$ has character $\geq m$. If not, let $x \in X$ be such that $x$ is not isolated and has a fundamental system of neighborhoods $\Re$ with $|\Re|<m$. We get a contradiction by showing that $H=X \backslash\{x\}$ is " $\Omega$-net closed" but clearly $H$ is not closed, If $D \in \Omega$ and $f: D \rightarrow H$, then $f$ does not converge to $x$, since, if it did, then for every $N \in \Re$ there would be $d_{N} \in D$ such that $d \geq d_{N}$ implies $f(d) \in N$. Let $d$ be an upper bound for $\left\{d_{N} \mid N \in \mathcal{N}\right\}$. Then $f(d)=x$ which contradicts the fact that the range of $f$ is in $H$. This shows that every convergent $\Omega$-net in $H$ converges to a point in $H$.

Example. A space $Y$ and a set $\Omega$ such that $Y$ satisfies (vii) of Proposition 1 , but $Y$ is not an $\Omega$-net space. Take $Y$ to be the convergent sequence $\{1 / n: n=1,2, \ldots\} \cup\{0\}$ with the usual topology, and $\Omega=\left\{\omega_{1}\right\}$. By the above lemma applied for $m=\omega_{1}, Y$ is not an $\left\{\omega_{1}\right\}$-net space. On the other hand, $Y$ does satisfy property (vii) since there is a convergent $\left\{\omega_{1}\right\}$-net $f$ such that $Y=W\left(f, \omega_{1}, 0\right)$.

We also point out that condition 6 of Theorem 1 is not equivalent for every $\Omega$ to the other conditions in Theorem 1 . The space $Y$ above has the $\left\{\omega_{1}\right\}$-weak topology, and $X$, the natural numbers with the discrete topology, is $\left\{\omega_{1}\right\}$-compact, but the projection from $X \times Y$ onto $Y$ is not 
closed. This also shows that $Y$ is not a subspace of any $\left\{\omega_{1}\right\}$-net space.

3. Infinite products. A class $\mathcal{C}$ of topological spaces is called an $\Omega$ compact test class if the following holds: For a space $X$, if the projection from $X \times Y$ to $Y$ is closed, for all $Y \in \mathcal{C}$, then $X$ is $\Omega$-compact. By Theorem 1 , the classes of $\Omega$-neighborhood, $\Omega$-Fréchet, $\Omega$-net spaces, and spaces with the $\Omega$-weak topology are $\Omega$-compact test classes. We agree that the product over an empty index set is the space consisting of one element.

Lemma (Noble [13, Theorem 1.8]). Let $X=\Pi\left\{X_{\alpha} \mid \alpha<m\right\}$ where $m$ is infinite. If for each $a_{0}<m$, the projection from $\Pi\left\{X_{\alpha} \mid \alpha \leq \alpha_{0}\right\}$ onto $\Pi\left\{X_{a} \mid a<a_{0}\right\}$ is closed, then for each $a_{0}$, the projection from $X$ onto $\Pi$ II $\left.X_{a} \mid \alpha<a_{0}\right\}$ is closed.

Theorem 2. Let $\Omega$ be a class of directed sets, $m$ an infinite cardinal, $\mathcal{C}$ an $\Omega$-compact test class, and $\left\{X_{\alpha} \mid \alpha<m\right\}$ a family of spaces. If for every $\alpha_{0}<m$ and for every $Y \in \mathcal{C}$, the projection from $\Pi\left\{X_{a} \mid \alpha \leq \alpha_{0}\right\} \times Y$ to $\Pi\left\{X_{a} \mid \alpha<a_{0}\right\} \times Y$ is closed, then $\Pi\left\{X_{a} \mid a<m\right\}$ is $\Omega$-compact.

Proof. To show that $X=\Pi\left\{X_{a} \mid a<m\right\}$ is $\Omega$-compact it suffices to take an arbitrary $Y \in \mathcal{C}$ and show that the projection from $X \times Y$ to $Y$ is closed. Define

$$
Z_{\alpha}= \begin{cases}Y & \text { if } \alpha=0, \\ X_{a-1} & \text { if } 0<a<\omega, \\ X_{a} & \text { if } \omega \leq \alpha<m .\end{cases}
$$

The hypothesis implies that the projection from $\operatorname{Il}\left\{Z_{a} \mid \alpha \leq \alpha_{0}\right\}$ onto $\Pi\left\{Z_{a} \mid a<\alpha_{0}\right\}$ is closed for each $\alpha_{0}<m$. By the above lemma, the projection from $\Pi\left\{Z_{a} \mid a<m\right\}$ onto $Z_{0}$ is closed, i.e., $\pi_{Y}: X \times Y \rightarrow Y$ is closed. Thus $X$ is $\Omega$-compact.

We now give a useful corollary to Theorem 2, and call attention to the fact that it reduces the question "When is the product of no more than $\mathrm{m}$ $\Omega$-compact, $\Omega$-neighborhood spaces again $\Omega$-compact?" to the question "When is the product of less than $\mathrm{m} \Omega$-neighborhood spaces again an $\Omega$-neighborhood space?".

Corollary 1. Let $\left\{X_{a} \mid \alpha<m\right\}$ be a family of $\Omega$-compact, $\Omega$-neighborhood spaces. If the product of less than $m \Omega$-neighborhood spaces is again an $\Omega$-neighborhood space, then $\Pi\left\{X_{a} \mid \alpha<m\right\}$ is $\Omega$-compact.

Proof. If $Y$ is an $\Omega$-neighborhood space, then so is $\Pi\left\{X_{a} \mid \alpha<\alpha_{0}\right\} \times Y$ for each $\alpha_{0}<m$. By Theorem 1, the projection from $X_{a_{0}} \times\left(\Pi\left\{X X_{a} \mid a<a_{0}\right\}\right.$ $\times Y)$ onto $\Pi\left\{X_{a} \mid \alpha<a_{0}\right\} \times Y$ is closed; so by Theorem 2, II $\left\{X_{a} \mid \alpha<m\right\}$ is $\Omega$-compact. 
From Corollary 1, we get the results of Noble that a countable product of Lindelöf $P$-spaces is Lindelöf, and that a product of no more than $m$ initially $m$-compact spaces of character $\leq m$ is initially $m$-compact. The idea to use closed projections to prove these product theorems is due to Noble. This last result can be proved using filters, and the referee pointed out that this was first done by I. Glicksberg [19, p. 380], who proved the following more general result: A product of at most $m$ initially $m$-compact spaces, all but at most one having character $\leq m$, is initially $m$-compact. Some recent developments in such product theorems are given in [20].

Noble's Theorem 4.1 in [13] can be restated as follows:

Corollary 2. If $X=\Pi\left\{X_{i} \mid i<\omega\right\}$ is a countable product where each $X_{i}$ is <a-discrete, of character $\leq \mathfrak{b}$, and satisfies property $G[a, b]$, then $X$ satisfies property $G[a, b]$.

Proof. Take $\Omega$ to be the class of all < $a$-directed sets of cardinality $\leq b$. A space is an $\Omega$-neighborhood space if and only if it is $<a$-discrete (i.e., every intersection of less than $a$ open sets is open) and has character $\leq \mathfrak{b}$. Since every finite product of such spaces is again a space of the same kind, the result follows from Corollary 1.

If we strengthen the hypothesis of Corollary 2 by making the assumption that each $X_{i}$ is $[a, b]$-compact, we still cannot conclude that the product $X$ is $[a, b]$-compact (see Example 3.6 of [16]). One way to get that conclusion is to require that $a$ be a regular cardinal and that $b^{\mathfrak{a}}=\mathfrak{b}$. With the se assumptions, $[a, b]$-compactness and property $G[a, b]$ are equivalent (see $[17$, The orem 2]).

\section{REFERENCES}

1. A. V. Arhangel'skii and S. P. Franklin, Ordinal invariants for topological spaces, Michigan Math. J. 15 (1968), 313-320; addendum, ibid. 15 (1968), 506. MR $39 \sharp 2112$.

2. Garrett Birkhoff, Moore-Smith convergence in general topology, Ann. of Math. 38 (1937), 39-56.

3. I. Fleischer and S. P. Franklin, On compactness and projection, Intenationale Spezialtagung für Erweiterungs-theorie topologischer Strukturen und deren Anwendunge, Berlin, 1967, pp. 77-79.

4. S. P. Franklin, Spaces in which sequences suffice, Fund. Math. 57 (1965), 107-115. MR $31 \# 5184$.

5. - Natural covers, Compositio Math. 21 (1969), 253-261. MR 40 \#3491.

6. D. Harris, Transfinite metrics, sequences and topological properties, Fund. Math. 73 (1971/72), 137-142. MR 46 \#864.

7. Horst Herrlich, Quotienten geordneter Räume und Folgenkonvergenz, Fund. Math. 61 (1967), 79-81. MR 36 \#4528. 
8. R. E. Hodel and J. E. Vaughan, A note on [a, b]-compactness, General Topology and Appl. 4 (1974), 179-189.

9. N. Howes, Well-ordered sequences, Dissertation, Texas Christian University, Forth Worth, Tex., 1968.

10. - Ordered coverings and their relationship to some unsolved problems in topology, Proc. Washington State University Conference on General Topology, 1970, pp. 60-68.

11. J. L. Kelley, General topology, Van Nostrand, Princeton, N. J., 1955. MR 16, 1136.

12. D. C. Kent, Spaces in which well ordered nets suffice, Proc. Washington State Univ. Conf. on General Topology (Pullman, Wash., 1970), Pi Mu Epsilon, Dept. of Math., Washington State Univ., Pullman, Wash., 1970, pp. 87-101. MR $42 \# 2419$.

13. N. Noble, Products with closed projections. II, Trans. Amer. Math. Soc. 160 (1971), 169-183. MR 44 \#979.

14. P. R. Meyer, Sequential space methods in general topological spaces, Colloq. Math. 22 (1971), 223-228. MR $43 \# 3989$.

15. S. Mrówka, On function spaces, Fund. Math. 45 (1958), 273-282. MR $20 \sharp 4773$.

16. J. E. Vaughan, Product spaces with compactness-like properties, Duke Math. J. 39 (1972), 611-617. MR $47 \# 2537$.

17. - Some properties related to $[\mathbf{a}, \mathbf{b}]$-compactness, Fund. Math. (to appear).

18. Karel Wichterle, $O_{n}$ B-convergence spaces, Czechoslovak Math. J. (18) 93 (1968), 569-588. MR $39 \sharp 2116$.

19. I. Glicksberg, Stone-ČCech compactifications of products, Trans. Amer. Math. Soc. 90 (1959), 369-382. MR $21 \# 4405$.

20. R. M. Stephenson, Jr. and J. E. Vaughan, Products of initially m-compact spaces, Trans. Amer. Math. Soc. 196 (1974), 177-189.

DEPARTMENT OF MATHEMATICS, UNIVERSITY OF NORTH CAROLINA AT GREENSBORO, GREENSBORO, NORTH CAROLINA 27412 